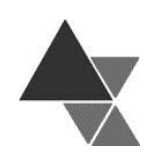

\title{
Aspectos nutricionais e qualidade de vida após cirurgia bariátrica em pacientes da Atenção Primária à Saúde
}

\author{
Manoela Nobrega Lorenzi ${ }^{1}$, Francini Xavier Rossetti ${ }^{2}$ e Karyne Sant'Ana Gonzales Gomes ${ }^{3}$
}

O trabalho tem como objetivo avaliar aspectos nutricionaisapós cirurgia bariátrica em pacientes da Atenção Primária à Saúde. Trata-se de estudo transversal exploratório com 30 pacientes cadastrados em quatro unidades de saúde de Curitiba (Paraná) submetidos à cirurgia bariátrica com período pós-operatório de 3 a 5 anos. Foram identificados dados sociodemográficos, clínico-cirúrgicos, antropométricos e aplicado o Método BAROS. A associação entre sucesso cirúrgico e idade, peso e índice de massa corporal pré-operatórios e atuais, uso de suplementos e realização de tratamento clínico anterior a cirurgia foram verificados por meio dos testes Mann Whitney, ANOVA e Teste G. Todos os pacientes realizaram gastroplastia com derivação intestinal. A média de idade foi 41,2 anos $(\mathrm{DP}=7,24)$, mulheres totalizaram 90\% dos entrevistados. Os valores relativos ao índice de massa corporal médio pré-operatório e atual foram $47 \mathrm{~kg} / \mathrm{m}^{2} \quad(\mathrm{DP}=7,43)$ e $32,25 \mathrm{~kg} / \mathrm{m}^{2} \quad(\mathrm{DP}=$ 6,46),respectivamente. Percentual de perda do excesso de peso médio alcançou 70,27\% (DP= 23,86). O insucesso terapêutico foi observado em sete $(23,4 \%)$ pacientes e dois $(6,6 \%)$ apresentaram resultado insuficiente. Ações de promoção, prevenção e tratamento precoce, desenvolvidas e ordenadas pela Atenção Primária em Saúde com participação de atores em articulação intersetorial - partindo da concepção de práticas alimentares saudáveis no contexto da Segurança Alimentar e Nutricional, podem reduzir a necessidade de cirurgias para tratamento da obesidade.

Palavras chave: obesidade mórbida, cirurgia bariátrica, Atenção Primária à Saúde.

\section{Nutritional aspects and quality of life after bariatric surgery in patients of Primary Health Care}

This paper aimed to evaluate the nutritional aspects of patients subjected to bariatric surgery in the primary health care program. An exploratory cross-sectional study was performed with 30 patients from four health care programs at Curitiba- PR subjected to bariatric surgery with postoperative period of 3 to 5 years. Sociodemographic, clinical- surgical, and anthropometric data were evaluated using BAROS method. The association between surgical success and age, weight, and preoperative and current body mass index, use of supplements, and previous clinical treatment were evaluated through the Mann Whitney test, ANOVA, and G Test. All

\footnotetext{
${ }^{1}$ Especialista pelo Programa de Residência Muiltiprofissional em Saúde da Família da Secretaria Municipal de Saúde de Curitiba. Graduada em Nutrição pela Universidade Federal do Paraná. Telefone: (41) 9982-4181.E-mail: manoelalorenzi@yahoo.com.br

2Professora das Faculdades Integradas do Vale do Ribeira (FVR/UNISEPE), Doutoranda em Ciência e Tecnologia de Alimentos, Escola Superior de Agricultura "Luiz de Queiroz", Universidade de São Paulo (ESALQ/USP). Endereço para correspondência: Rua Oscar Y. Magárion॰ 185, Jardim das Palmeiras, Registro, SP, CEP: 11900-000. Email:francini@usp.br

${ }^{3}$ Especialista em Saúde Coletiva pela Associação Brasileira de Alimentação e Nutrição. Nutricionista do Núcleo de Apoio à Saúde da Família da Secretaria Municipal de Saúde de Curitiba, PR, Brasil. Telefone: (41) 9977-7459.E-mail:karynut@hotmail.com
} 
patients underwent gastric intestinal bypass. The mean age was 41.2 years $(\mathrm{SD}=7.24), 90 \%$ female. The mean preoperative and current body mass index were $47 \mathrm{~kg} / \mathrm{m}^{2}(\mathrm{SD}=7.43)$ and $32.25 \mathrm{~kg} / \mathrm{m}^{2}(\mathrm{SD}=6.46)$ respectively. The mean excess weight loss was $70.27 \%(\mathrm{SD}=23.86)$. Therapeutic failure was observed in seven $(23.4 \%)$ patients, and insufficient results by BAROS method in two $(6.6 \%)$ patients. Actions on health promotion, prevention, and early treatment by the primary health care program can reduce the need for surgical treatment of obesity, with the participation of actors in intersectorial articulation, considering the healthy food practices in the context of Food and Nutrition Security.

Keywords: morbid obesity, bariatric surgery, Primary Health Care.

\section{INTRODUÇÃO}

O excesso de peso e comorbidades associadas constituem importante problema de saúde pública. No Brasil, dados de 2014 apontam 52,5\% da população adulta com excesso de peso (sobrepeso e obesidade), sendo $17,9 \%$ com obesidade. O município de Curitiba - PR segue padrão nacional sendo que $53,8 \%$ da população apresenta excesso de peso e 18,8\% obesidade ${ }^{[1,2]}$.

O excesso de peso tem como determinantes proximais o padrão alimentar e o dispêndio energético. Pesquisas nacionais identificam na população brasileira um elevado consumo de alimentos ricos em açúcar, gorduras saturadas, trans e sal e baixo consumo de carboidratos complexos e fibras. A diminuição da prevalência de desnutrição e o aumento do sobrepeso e obesidade evidenciam o fenômeno da transição nutricional [2,3].

A prevenção e tratamento da obesidade são grandes desafios para o Sistema Único de Saúde (SUS). Estratégias devem estar contidas nas redes de atenção, em especial na Rede de Atenção à Saúde das Pessoas com Doenças Crônicas, com a organização da Linha de Cuidado da Obesidade em cada Região de Saúde, prevendo conectividade e integração entre os diferentes pontos de atenção [2].

O tratamento cirúrgico consiste em parte do tratamento integral da obesidade, que deve ser prioritariamente centrado na promoção da saúde e no cuidado clínico longitudinal [5]. Ações de promoção, prevenção e tratamento precoce, baseados em mudança de hábitos de vida, que podem ser desenvolvidos e ordenados pela Atenção Primária em Saúde com participação de atores em articulação intersetorial, partindo da concepção de Segurança
Alimentar e Nutricional podem reduzir a necessidade da realização de intervenções cirúrgicas para o tratamento da obesidade.

No Brasil, a cirurgia inclui-se entre os procedimentos de alta complexidade cobertos pelo SUS desde 1999. Entre 1999 e 2006 foram realizadas mais de 10.000 cirurgias bariátricas pelo SUS [6,7]. Em 2010 foram realizadas 64 mil cirurgias no país nos setores público e privado, uma alta de $275 \% \mathrm{em}$ relação a 2003. O país é o segundo no ranking mundial de cirurgias bariátricas, perdendo apenas para os Estados Unidos [8]. Em Curitiba, entre janeiro de 2010 e dezembro de 2012 foram realizadas 2014 cirurgias, sendo $1920(95,3 \%)$ gastroplastias com derivação intestinal [9].

A alta da assistência pós-operatória no nível de atenção de alta complexidade deve ocorrer no mínimo 18 meses após a intervenção cirúrgica, quando é preconizado encaminhamento para o nível primário de atenção [3]. Neste contexto, o objetivo do presente trabalho é avaliar aspectos nutricionaise qualidade de vida após cirurgia bariátrica em pacientes da Atenção Primária à Saúde.

\section{MÉTODOS}

Trata-se de estudo transversal e exploratório, de abordagem quantitativa. Foram entrevistados pacientes residentes na área de abrangência de quatro Unidades de Saúde que ofertam cuidados de Atenção Primária à Saúde do município de Curitiba - PR. Todos foram submetidos à cirurgia bariátrica custeada pelo SUS, realizadas em sete hospitais prestadores, contando com 3 a 5 anos de pós-operatório. 
Para a inclusão no estudo foram considerados pacientes de ambos os sexos, idade superior a $18 \mathrm{e}$ inferior a 65 anos, que realizaram umdos procedimentos: gastrectomia com ou sem desvio duodenal, gastrectomia vertical em manga - sleeve, gastroplastia com derivação intestinal ou gastroplastia vertical com banda. Os indivíduos que integraram a pesquisa foram entrevistados após concordarem em participar e mediante assinatura do Termo de Consentimento Livre e Esclarecido. Foram excluídos os usuários que realizaram a cirurgia por meio de convênio ou particular.

A amostra foi obtida por conveniência. A identificação e abordagem dos participantes foram realizadas nas Unidades de Saúde de quatro modos: 1) ao buscar atendimento na unidade; 2) ao agendar consulta com a Nutricionista ${ }^{1}$; 3 ) por encaminhamento da equipe de saúde e 4) chamamento público com cartazes afixados nas unidades.

As entrevistas foram realizadas entre novembro e dezembro de 2015. O estudo foi aprovado nos Comitês de Ética e Pesquisa das Faculdades Pequeno Príncipe (parecer 1.143.219) e da Secretaria Municipal de Saúde de Curitiba (protocolo 90/2015).

Foram identificados dados sociodemográficos (sexo, idade, estado civil, atividade laboral, unidade de saúde que estava cadastrado) e clínico-cirúrgicos (tratamentos para perda de peso anteriores à cirurgia, uso atual de suplementação vitamínica e/ou mineral e realização de reposição de cobalamina injetável).

Foi aplicado o Método BAROS (Bariatric Analysis and Reporting Outcome System) segundo Oria e Moorehead, instrumento de avaliação da cirurgia bariátrica que analisa perda de peso, mudanças nas comorbidades e qualidade de vida. O sistema define cinco resultados possíveis: insuficiente, aceitável, bom, muito bom e excelente ${ }^{10]}$. O questionário incorporado ao BAROS foi o Moorehead-Ardelt Quality of Life Questionnaire II que avalia auto-estima, realização de atividade física, contatos sociais, desempenho no trabalho, prazer em afetividade e/ou sexo e relação com a comida ${ }^{[1]}$.
Foi realizada avaliação antropométrica atual com aferição do peso utilizando a balança eletrônica Marte ${ }^{\circledR}$, Modelo PP180, com sensibilidade de 0,1 kg e a estatura por meio de estadiômentro acoplado à parede com precisão de $0,1 \mathrm{~cm}$. Foi calculado peso ideal, considerando a altura aferida e o Índice de Massa Corporal (IMC) $25 \mathrm{~kg} / \mathrm{m}^{2}$. O excesso de peso foi calculado subtraindo o peso ideal do peso préoperatório. Para a avaliação da efetividade do tratamento cirúrgico da obesidade foi utilizado o percentual de perda do excesso de peso (\%PEP) considerando sucesso terapêutico uma perda superior a $50 \%$ do excesso de peso, e um excelente resultado uma perda superior a $75 \%$ do excesso de peso.

Os dados foram tabulados por meio dos recursos do Microsoft Office Excel Start. Testes estatísticos foram realizados com o auxílio do software Bioestat $5.0^{\circledR}$. Para verificação da aderência aos parâmetros de normalidade foi utilizado o teste Liliefors. Para a análise dos dados contínuos não paramétricos foi utilizado o teste de Mann Witney. Para os dados contínuos paramétricos foi utilizado ANOVA, seguida do teste de Tukey. As comparações das distribuições proporcionais entre os grupos foram realizadas por meio do Teste G. A significância estatística considerada foi de $5 \%(\phi \leq 0,05)$ para todas as análises realizadas.

\section{RESULTADOS}

O peso e o IMC pré-operatórios médios encontrados foram de $124,11 \mathrm{~kg}(\mathrm{DP}=24,07) \mathrm{e}$ $47 \mathrm{~kg} / \mathrm{m}^{2}$ (DP= 7,43)respectivamente. A média do excesso de peso anterior à realização da cirurgia foi de $58,8 \mathrm{~kg}(\mathrm{DP}=21,35)$. Os pesos e IMC médios atuais aferidos durante a entrevista foram respectivamente $83,68 \mathrm{~kg}(\mathrm{DP}=15,32)$ e $32,25 \mathrm{~kg} / \mathrm{m}^{2}(\mathrm{DP}=6,46) . \mathrm{A}$ gastroplastia com derivação intestinal foi referida por todos os participantes. As variáveis sociodemográficas estão dispostas na Tabela 1.

${ }^{1}$ Primeira autora do artigo 
Tabela 1. Características sociodemográficas e clínico-cirúrgicas. Curitiba (PR), 2015.

\begin{tabular}{|c|c|c|}
\hline Variáveis & $\mathrm{n}$ & $\%$ \\
\hline \multicolumn{3}{|l|}{ Sexo } \\
\hline Feminino & 27 & 90,0 \\
\hline Masculino & 03 & 10,0 \\
\hline \multicolumn{3}{|l|}{ Faixa etária } \\
\hline 20-35 anos & 08 & 26,7 \\
\hline 36-50 anos & 19 & 63,3 \\
\hline $51-65$ anos & 03 & 10,0 \\
\hline \multicolumn{3}{|l|}{ Estado conjugal } \\
\hline Com companheiro & 20 & 66,7 \\
\hline Sem companheiro & 10 & 33,3 \\
\hline \multicolumn{3}{|l|}{ Ocupação } \\
\hline Com remuneração & 27 & 90,0 \\
\hline Sem remuneração & 03 & 10,0 \\
\hline \multicolumn{3}{|c|}{ Índice de Massa Corporal pré-operatório $\left(\mathrm{kg} / \mathrm{m}^{2}\right)$} \\
\hline $35-39,9$ & 05 & 16,7 \\
\hline $40-49,9$ & 17 & 56,7 \\
\hline$\geq 50$ & 08 & 26,7 \\
\hline \multicolumn{3}{|c|}{ Tratamento para perda de peso pré-operatório ${ }^{1}$} \\
\hline Alimentar & 09 & 30,0 \\
\hline Medicamentoso & 16 & 53,3 \\
\hline Atividade física & 06 & 20,0 \\
\hline Não realizou & 03 & 10,0 \\
\hline \multicolumn{3}{|c|}{ Uso atual de suplementos minerais/vitamínicos } \\
\hline $\operatorname{Sim}$ & 14 & 46,7 \\
\hline Não & 16 & 53,3 \\
\hline \multicolumn{3}{|c|}{ Reposição vitamina B12 - injetável ${ }^{2}$} \\
\hline $\operatorname{Sim}$ & 11 & 36,7 \\
\hline Não & 19 & 63,3 \\
\hline
\end{tabular}

${ }^{1}$ Considerado período mínimo de um ano.

${ }^{2}$ Pelo menos uma vez após a cirurgia.

O \%PEP médio foi de 70,27\% (DP= 23,86). Atingiram o critério utilizado para a classificação do sucesso do tratamento cirúrgico (PEP $\geq 50 \%$ ), 23 $(76,6 \%) \quad$ entrevistados. Destes, $17 \quad(56,6 \%)$ apresentaram uma perda de peso superior a $75 \%$ do excesso de peso (PEP>75\%), o que pode ser considerado um excelente resultado. A Tabela 2 apresenta as variáveis antropométricas relacionadas ao resultado encontrado na aplicação do método BAROS. 
Tabela 2. Variáveis antropométricas e idade conforme resultado da aplicação do BAROS. Curitiba (PR), 2015.

\begin{tabular}{cccccc}
\hline Classificação & $\begin{array}{c}\text { Pacientes } \\
\text { (n) }\end{array}$ & $\begin{array}{c}\text { Idade média } \\
\text { (DP) }\end{array}$ & $\begin{array}{c}\text { IMC médio pré } \\
\text { cirurgia (DP) }\end{array}$ & $\begin{array}{c}\text { IMC médio pós } \\
\text { cirurgia (DP) }\end{array}$ & $\begin{array}{c}\text { \% de perda de } \\
\text { peso médio } \\
\text { (DP) }\end{array}$ \\
\hline Insuficiente & 2 & $44,5(3,5)$ & $45,0(1,4)$ & $39,5(2,1)$ & $29,0(7,1)$ \\
Aceitável & 3 & $42,3(12,2)$ & $48,0(2,6)$ & $37,3(6,0)$ & $46,3(21,5)$ \\
Bom & 10 & $40,7(7,7)$ & $49,9(8,8)$ & $33,5(7,6)$ & $65,1(21,9)$ \\
Muito bom & 6 & $39,7(4,3)$ & $46,8(6,5)$ & $31,2(4,8)$ & $72,2(16,4)$ \\
Excelente & 9 & $41,7(8,2)$ & $46,8(9,2)$ & $27,4(3,4)$ & $89,1(11,0)$ \\
\hline
\end{tabular}

A Tabela 3 apresenta os indivíduos categorizados com $\% \mathrm{PEP}<50 \%$ ou $\geq 50 \%$ e comparação entre as variáveis antropométricas e sociodemográficas. Foi encontrada diferença estatística entre os grupos para o peso atual, o IMC atual e para o uso atual de suplementos.

Tabela 3. Variáveis distribuídas conforme a perda do excesso de peso (\%PEP). Curitiba (PR), 2015.

\begin{tabular}{|c|c|c|c|}
\hline Variáveis & $\begin{array}{c}\% \text { PEP } \\
<50 \% \\
\mathrm{n}=7 \text { (DP) } \\
\end{array}$ & $\begin{array}{c}\% \text { PEP } \\
\geq 50 \% \\
\mathrm{n}=23 \text { (DP) }\end{array}$ & $P$ \\
\hline Idade & $44,1(5,8)$ & $40,3(7,5)$ & $0,223^{1}$ \\
\hline Peso pré-cirúrgico & $121,0(10,1)$ & $125,0(27)$ & $0,731^{2}$ \\
\hline IMC pré-cirúrgico & $49,7(4,6)$ & $46,8(7,8)$ & $0,619^{1}$ \\
\hline Excesso de peso & $60,0(9,5)$ & $58,4(24)$ & $0,249^{2}$ \\
\hline Peso atual & $99,3(8,9)$ & $78,9(13,6)$ & $<0,01^{1}$ \\
\hline IMC atual & $40,7(3,2)$ & $29,7(4,7)$ & $<0,01^{1}$ \\
\hline
\end{tabular}

${ }^{1}$ Tukey após ANOVA $(p \leq 0,05)$

${ }^{2}$ Mann-Witney

${ }^{3}$ Teste $\mathrm{G}$

\section{DISCUSSÃO}

A gastroplastia com derivação intestinal consiste em procedimento misto que combina componente restritivo e disabsortivo [5,12]. A perda de peso ocorre por fatores mecânicos e hormonais, com a redução da ingestão alimentar em decorrência de uma menor capacidade gástrica e do apetite pela redução da grelina sérica (hormônio orexígeno) e aumento do peptídeo Y e glucagon-like peptídeo 1 (GLP1) (hormônios anorexígenos). Associa-se ainda a redução da absorção de nutrientes [12,13,14]. Técnicas mistas constituem padrão ouro de operações para obesidade grave, relacionadas à melhora das comorbidades, qualidade de vida e manutenção de resultados a longo prazo, sendo a opção atualmente mais utilizada pelos cirurgiões [7,14,15,16]. Foi a técnica adotada para os procedimentos envolvendo a totalidade dos os participantes da presente pesquisa.
Ainda que os benefícios da cirurgia bariátrica sejam amplamente relatados na literatura, 2 a 3 anos após o procedimento, o paciente passa a sofrer os efeitos secundários com menor intensidade, observando-se a redução da síndrome de dumping, dilatação do reservatório gástrico, aumento do calibre da anastomose gastrojejunal e adaptações hormonais e fisiológicas do trato gastrointestinal [6,12,13,14,15].

Da mesma forma, fatores não cirúrgicos podem contribuir para a recuperação do peso, como hábitos alimentares inadequados, sedentarismo, baixa adesão ao acompanhamento pós-operatório, índice de massa corporal inicial muito elevado, entre outras [12,15].

O procedimento é procurado predominantemente por mulheres, uma vez que para estas o controle de peso tem uma forte pressão de motivação estética, além de importantes componentes 
emocionais em relação à comida [6,15,17]. As mulheres também apresentam uma maior prevalência de obesidade mórbida no Brasil quando analisados dados populacionais, chegando a 3\% dos adultos de ambos os sexos com 18 anos ou mais, sendo $2 \%$ em homense $4 \%$ em mulheres $[6,12,15]$. No presente estudo, as mulheres totalizaram $27(90 \%)$ dos indivíduos entrevistados.

A gastroplastia com derivação intestinal induz a uma média de perda de 60 a 75\% do excesso de peso corporal [6,15], semelhante ao resultado encontrado no presente estudo, que foi de 70,29\% (DP=23,86).

A idade, o peso e o IMC pré-cirúrgicos não apresentaram associação em relação ao sucesso do tratamento (Tabela 3). Apenas o peso e o IMC atuais apresentaram associação significativa $(\phi<0,05)$, semelhante ao encontrado por Novais et al. [13].

A literatura científica recomenda diversos critérios para avaliação da efetividade do tratamento cirúrgico da obesidade Uma classificação recorrente para o sucesso é um \% $\%$ EP $<50[13,15,17]$. Pode ainda ser considerado um excelente resultado um \% $\%$ EP $>75$ [12].

Um $\% \mathrm{PEP}<50 \%$ foi encontrado em sete $(23,4 \%)$ participantes da pesquisa, considerado insucesso no tratamento. Bastos et al. ${ }^{[15]}$ avaliaram 64 pacientes e identificaram uma falha de 6,25\%, enquanto Farias [17], ao avaliar 204 indivíduos encontrou uma falha de 59 (28,9\%). Valezi et al. [14] encontraram insucesso em $7,1 \%$ de 134 pacientes. Todos os estudos avaliaram técnicas mistas de tratamento cirúrgico da obesidade em período superior a 2 anos da realização da cirurgia.

Apesar de ser um indicador importante, o peso e a perda de peso não devem ser tomados isoladamente na avaliação do resultado, uma vez que outros benefícios podem ser encontrados, seja em qualidade de vida ou melhora das comorbidades associadas [8,10,16]. O método BAROS é uma avaliação que leva em consideração estes aspectos, além de possíveis complicações advindas do procedimento cirúrgico [10,11]. A aplicação do BAROS encontrou um resultado bom, muito bom e excelente para 25 indivíduos $(83,3 \%)$, o que demonstra um resultado importante na melhora da qualidade de vida, superior ao encontrado para o sucesso cirúrgico considerado a perda de peso isoladamente. Prevedello et al. [6] encontraram este resultado para os 32 pacientes para os quais foi aplicado o método BAROS. Bastos et al. [15] observaram este resultado para 95,3\% e Farias [17] para $79,49 \%$ dos 156 pacientes entrevistados.

O acompanhamento pós-operatório deve contemplar exames bioquímicos, inquéritos dietéticos e exames físicos individualizados. A realização da cirurgia bariátrica frequentemente conduz à deficiência de micronutrientes e alterações hematológicas devido à insuficiência de ingestão ou absorção de ferro, folato e cobalamina [6,8,15]. O uso de suplementos minerais/vitamínicos é uma opção frequente para atingir as recomendações diárias ${ }^{[16] . ~}$

O presente estudo encontrou uso regular de suplementos em $14 \quad(46,6 \%)$ dos pacientes entrevistados, apresentando correlação estatisticamente significativa com o sucesso do tratamento cirúrgico através da realização do Teste $G$ $(p<0,05)$. Tal correlação pode indicar que estes pacientes tem preocupação maior com a saúde e modificaram padrões de comportamento. Bastos et al. [15] constataram uso de suplementos em 73,4\% dos 64 indivíduos entrevistados enquanto Farias ${ }^{[17]}$ encontrou a suplementação regular em 69,1\% dos 204 indivíduos. Tal resultado pode ter relação com o fato desses estudos citados terem sido realizados em ambiente hospitalar, por pesquisadores com relação direta com a equipe cirúrgica, ao contrário do presente estudo que foi realizado na Atenção Primária em Saúde, cuja responsabilidade do seguimento pós-cirúrgico ainda não está consolidada.

A indicação cirúrgica leva em consideração idade, IMC prévio em faixa de risco há pelo menos dois anos, presença de comorbidades e realização de tratamentos convencionais prévios com insucesso ou recidiva no peso $[16,18,19]$.

Para realização da cirurgia pelo SUS, o tempo estabelecido para o tratamento clínico em Atenção Primária ou Atenção Especializada para o IMC acima de 35 e abaixo de $50 \mathrm{~kg} / \mathrm{m}^{2}$ anteriores ao encaminhamento para o serviço de Alta Complexidade é de pelo menos dois anos [4,5,19]. Encontrou-se neste estudo que 3 indivíduos (10\%) nunca realizaram qualquer tratamento clínico. $\mathrm{O}$ tratamento nutricional por pelo menos um ano antes do encaminhamento para a cirurgia foi realizado por $9(30 \%)$ entrevistados, no tocante à utilização de medicamentos $16(53,3 \%)$ 
indivíduos referiram uso e por fim, $6(20 \%)$ destes referiram prática de atividade física como método de tratamento para a perda ponderal anterior ao tratamento cirúrgico. De maneira geral, tal realidade indica que tratamentos clínicos com foco na mudança de hábitos de vida não precederam o encaminhamento para o serviço de alta complexidade.

\section{CONCLUSÃO}

O percentual médio de perda do excesso de peso de $70 \%(\mathrm{DP}=23,86)$ demonstra que, se por um lado, a gastroplastia com derivação intestinal atingiu o esperado para a técnica cirúrgica e período de pósoperatório, por outro lado, apresentou um importante insucesso cirúrgico de $23,4 \%$ dos indivíduos entrevistados.

O crescente número de cirurgias realizadas e o resultado encontrado reforça a necessidade de qualificar a continuidade do atendimento, que após 18 meses de cirurgia deve ocorrer na Atenção Primária em Saúde.

Também deve ser consolidada a Linha de Cuidado da Obesidade, garantindo a assistência integral à saúde do indivíduo obeso em todos os níveis de atenção, priorizando a promoção de hábitos de vida saudáveis e tornando o tratamento cirúrgico a opção apenas para os casos mais graves e aqueles cujo tratamento clínico resultou em insucesso.

\section{REFERÊNCIAS}

[1] Ministério da Saúde. Vigitel Brasil 2014. Vigilância de fatores de risco e proteção para doenças crônicas por meio de inquérito telefônico. Brasília, DF: Ministério daSaúde, 2015.

[2] Brasil. Portaria no 483, de 1o de abril de 2014. Redefine a Rede de Atenção à Saúde das Pessoas com Doenças Crônicas no âmbito do Sistema Único de Saúde (SUS) e estabelece diretrizes para a organização de suas linhas de cuidado. Diário Oficial da União. 02 abr 2014; Seção 1.

[3] Ministério da Saúde. Guia alimentar para a população brasileira. Brasília, DF: Ministério da Saúde, 2014.

[4] Ministério da Saúde. Cadernos de Atenção Básica no 38: estratégias para o cuidado da pessoa com doença crônica Obesidade. Brasília, DF: Ministério da Saúde, 2014.

[5] Brasil. Portaria no 425, de 19 de março de 2013. Estabelece regulamento técnico, normas e critérios para o Serviço de
Assistência de Alta Complexidade ao Indivíduo com Obesidade. Diário Oficial da União. 15 abr 2013; Seção 1.

[6] Prevedello CF, Colpo E, Mayer ET, Copetti H. Análise do impacto da cirurgia bariátrica em uma população do centro do estado do Rio Grande do Sul utilizando o Método Baros. Arq Gastroenterol. 2009;46(3):199-203. DOI http://dx.doi.org/10.1590/S0004-28032009000300011.

[7] Scabim VM, Neto JE, Tess BH. Adesão ao seguimento nutricional ambulatorial pós-cirurgia bariátrica e fatores associados. Rev Nutr. 2012;25(4):497-506. DOI http://dx.doi.org/10.1590/S1415-52732012000400007.

[8] Marcelino LF, Patricio ZM. A complexidade da obesidade e o processo de viver após a cirurgia bariátrica: uma questão se saúde coletiva. Ciênc. Saúde Coletiva. 2011;16(12):4767-4776. DOI http://dx.doi.org/10.1590/S1413-81232011001300025.

[9] Brasil. Ministério da Saúde. Departamento de Informática do SUS. Procedimentos hospitalares por local de moradia [internet]. 2014. [acesso em 05 dez 2014]. Disponível em: $<$ http://tabnet.datasus.gov.br $>$.

[10] Oria HE, Moorehead MK. Bariatric Analysis and Reporting Outcome System (BAROS). Obes Surg. 1998;8:48799. DOI http://dx.doi.org/10.1381/096089298765554043.

[11] Oria HE, Moorehead MK. Updated Bariatric Analysis and Reporting Outcome System (BAROS). Surg Obes Relat Dis. 2009;5(1):60-6. DOI http://dx.doi.org/10.1016/j.soard.2008.10.004.

[12] Rocha QS, Mendonça SS, Fortes RC. Perda ponderal após gastroplastia em Y de Roux e importância do acompanhamento nutricional: uma revisão de literatura. Comun. Ciên. Saúde. 2011;22(1):61-70.

[13] Novais PFS, Junior IR, Leite CVS, Oliveira MRM. Evolução e classificação do peso corporal em relação aos resultados da cirurgia bariátrica - derivação gástrica em Y de Roux. Arq Bras Endocrinol Metab. 2010;54(3):303-310. DOI http://dx.doi.org/10.1590/S0004-27302010000300009.

[14] Valezi AC, Junior JM, Menezes MA, Brito EM, Souza JCL. Evolução ponderal oito anos após derivação gástrica em Y-deRoux. Rev. Col. Bras. Cir. 2011;38(4):232-236. DOI http://dx.doi.org/10.1590/S0100-69912011000400006.

[15] Bastos ECL, Barbosa EMWG, Soriano GMS, Santos EA, Vasconcelos SML. Fatores determinantes do reganho ponderal no pós-operatório de cirurgia bariátrica. Arqu. Bras. Cir. Dig. 2013;26(Suppl 1):26-32. DOI http://dx.doi.org/10.1590/S010267202013000600007 .

[16] Junior ABG, Ferraz EM, Barroso FL, Marchesini JB, Szegö T. Cirurgia da obesidade. São Paulo: Atheneu; 2006. 
[17] Farias G. Redução de peso após cirurgia bariátrica: diferenças clínicas e nutricionais entre bons e maus respondedores [dissertação]. Curitiba: Universidade Federal do Paraná; 2014.

[18] Sociedade Brasileira de Cirurgia Bariátrica. Consenso Bariátrico Brasileiro [internet]. 2006. [acesso em 20 jan 2015]. Disponível em: http://www.sbcb.org.br/arquivos/download/consenso_bariatrico. pdf.
[19] Brasil. Portaria no 424, de 19 de março de 2013. Redefine as diretrizes para a organização da prevenção e do tratamento do sobrepeso e obesidade como linha de cuidado prioritária da Rede de Atenção à Saúde das pessoas com Doenças Crônicas. Diário Oficial da União. 28 jun 2013; Seção 1. 